\title{
Welche gesetzliche Regelung braucht die patientenorientierte Forschung?
}

\author{
Die Umsetzung der ICH-GCP-Richtlinie führt bei vielen klinischen Studien zu Überre- \\ gulierung. Dies sollte im Rahmen des Humanforschungsgesetzes korrigiert werden. \\ Benötigt werden günstige Rahmenbedingungen für patientenorientierte Forschung.
}

\begin{abstract}
Peter Brauchli ,
Pascale Wenger ${ }^{b}$,

Beat Thürlimannc,

Thomas Cerny', Rolf Stahele,

Nicolas von der Weidf,

Jakob Passwegg,

Richard Herrmann ${ }^{h}$

Vor einiger Zeit hat Swissmedic eine Professionalisierung der klinischen Forschung gefordert, weil die Qualität von klinischen Studien in einigen Bereichen ungenügend sei [1]. Auf der Seite der Forschenden wird der Unmut gegenüber gewissen Anforderungen der Behörden (Ethikkommissionen und Swissmedic) bezüglich Bewilligungen, Notifikationen und Inspektionen immer lauter.
\end{abstract}

a Dr. sc. nat.,

Direktor Schweizerische Arbeitsgemeinschaft für klinische Krebsforschung SAKK, Geschäftsführer Oncosuisse

b Leiterin Regulatory Affairs SAKK

c Prof. Dr. med., Präsident SAKK

d Prof. Dr. med., Präsident Stiftung Krebsforschung Schweiz

e Prof. Dr. med., Präsident International Breast Cancer Study Group IBCSG, Präsident der European Thoracic Oncology Platform ETOP

f PD Dr. med., Präsident Schweizerische Pädiatrische Onkologie Gruppe SPOG

g Prof. Dr. med., Präsident Krebsliga Schweiz

h Prof. Dr. med., Präsident Oncosuisse

Korrespondenz:

Dr. sc. nat. Peter Brauchli

Effingerstrasse 40

CH-3008 Bern

Tel. 0313899191

peter.brauchli@sakk.ch

www.sakk.ch

www.oncosuisse.ch

\section{Einführung von Good Clinical Practice (GCP)}

Die Erkenntnis, dass klinische Forschung neben ethischen Richtlinien (Declaration of Helsinki, 1964) auch Verfahrensrichtlinien benötigt, reifte in den 70er Jahren. Das unbestrittene Ziel von GCP ist es, die Sicherheit und Rechte der Patienten sowie die Qualität der Daten zu garantieren. 1977 führten die USA als erstes Land GCP-Regeln ein. Die Schweiz führte das Reglement über die Heilmittel im klinischen Versuch inkl. Guter Praxis der Klinischen Versuche (GPKV) 1993 ein.

1996 verabschiedete die International Conference on Harmonisation of Technical Requirements for Registration of Pharmaceuticals for Human Use (ICH) mit Vertretern der Pharmazeutischen Industrie und Behörden aus den Regionen USA, EU und Japan die ICH-GCP Guideline E6. Darin heisst es: «The objective of this ICH GCP Guideline is to provide a unified standard for the European Union (EU), Japan and the United States to facilitate the mutual acceptance of clinical data by the regulatory authorities in these jurisdictions. (...) This guideline should be followed when generating clinical trial data that are intended to be submitted to regulatory authorities.» [2]

Diese Richtlinie ist auf die Medikamentenentwicklung der pharmazeutischen Industrie und damit auf die Durchführung von Studien mit neuen, nicht registrierten Medikamenten ausgerichtet.

In der Schweiz wurden 2002 das Heilmittelgesetz (HMG) und die Verordnung für Klinische Versuche (VKlin), mit der die Einhaltung der ICH-GCP-Richtlinie als verbindlich für alle Studien mit Medikamenten erklärt wird, in Kraft gesetzt. Daher wird in der Schweiz oft vereinfachend GCP mit der ICH-GCPzige Land in Europa, das diese (zu) detaillierte Richtlinie ins Gesetz integrierte. Die EU erkannte die Unzulänglichkeit eines solchen Schrittes und führte eine Richtlinie gleichgesetzt. Die Schweiz ist damit das ein-

\section{Quelle base légale pour la recher- che centrée sur le patient?}

La conduite d'essais cliniques est soumise à des règles définies, notamment d'ordre éthique (Bonnes Pratiques Cliniques). Avec l'entrée en vigueur de la Loi sur les produits thérapeutiques en 2002, la directive de I'ICH relative aux bonnes pratiques des essais cliniques est devenue le règlement pour toutes les études portant sur des produits thérapeutiques. L'application actuelle de cette directive a entraîné un excès de rigueur dans certaines études. Il convient donc d'apporter des corrections dans le cadre de la loi relative à la recherche sur l'être humain et d'établir des conditions générales favorables pour la recherche axée sur les patients.

eigene GCP-Richtlinie ein (Commission Directive 2005/28/EC).

\section{ICH-GCP wird der patientenorientierten Forschung nicht gerecht}

Ein Verdienst der Umsetzung von ICH-GCP ist sicher, dass qualitativ ungenügende Studienprojekte mit Medikamenten nicht mehr möglich sind. «Good Clinical Practice» wurde aber keineswegs mit dieser Richtlinie eingeführt, sondern war in der Schweiz bereits zuvor durch die GPKV adäquat geregelt. Wir möchten unmissverständlich festhalten, dass es für keine Art von klinischen Studien Abstriche an den GCP-Grundsätzen geben darf. Für verschiedene klinische Bereiche müssen aber die relevanten Detailanforderungen spezifiziert werden [3, 4]. Die heute praktizierte wortgetreue Umsetzung durch die Behörden führt zu Anforderungen, die wir als unverhältnismässig einschätzen.

In der Schweiz wird die ICH-GCP-Richtlinie auch für Forschungsbereiche angewendet, für die sie in keiner Weise ausgelegt wurde, wie zum Beispiel multimodale Studien, bei denen neben Medikamenten auch chirurgische und radiotherapeutische Verfahren ein- 


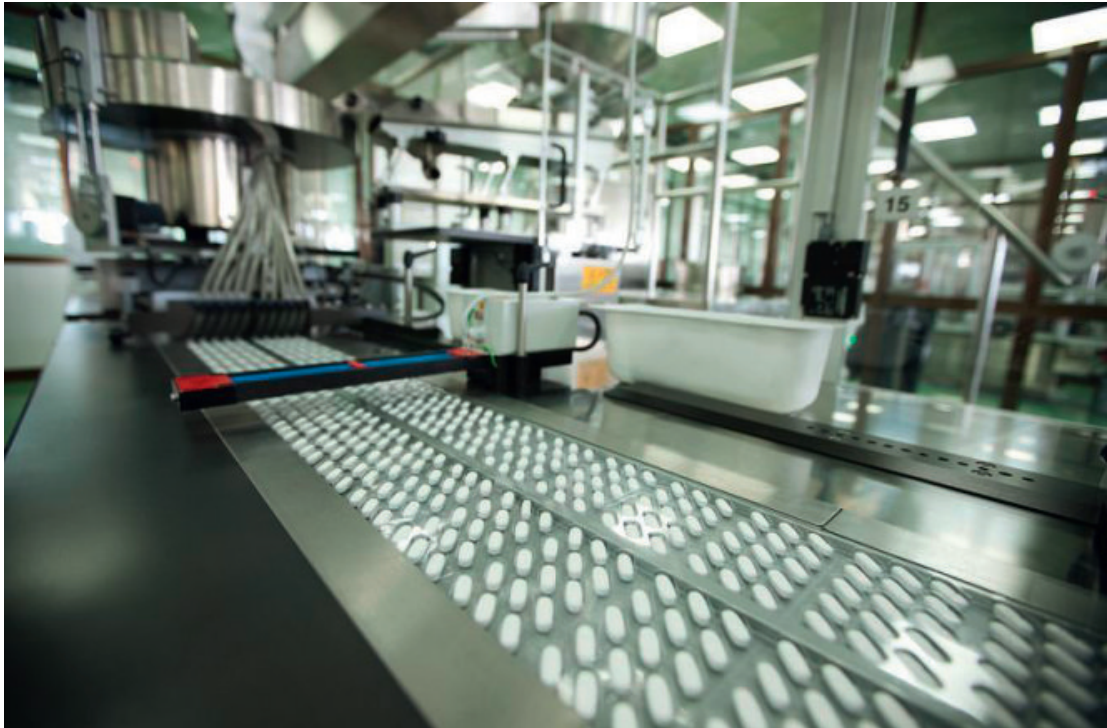

Die ICH-GCP-Richtlinie ist auf die Medikamentenentwicklung der pharmazeutischen Industrie, auf Studien mit neuen, nicht registrierten Medikamenten ausgerichtet. Anderen Forschungsbereichen wird sie nicht gerecht.

1 Baumann A. Swissmedic fordert Professionalisierung der Forschung. Schweiz Ärztezeitung. 2009;90(37) 1419.

2 International Conference on Harmonisation of Technical Requirements for Registration of Pharmaceuticals for Human Use GUIDELINE FOR GOOD CLINICAL PRACTICE E6(R1). p. 1. www.ich.org/ LOB/media/MEDIA482.pdf

3 Stewart DJ, Whitney SN, Kurzrock R. Equipoise Lost: Ethics, Costs, and the Regulation of Cancer Clinical Research. J Clin Oncol. 2010;28:2925-35.

4 Oncosuisse. Nationales Krebsprogramm 2011-2015. www.oncosuisse.ch

5 Bergmann L, Berns B, Dalgleish AG, von Euler M, Hecht TT, Lappin GL et al. Investigator-initiated trials of targeted oncology agents: why independent research is at risk? Ann Oncol. 2010;21;1573-8.

6 ECRIN. Road Map Initiative for Clinical Research in Europe. www.ecrin.org

7 ADAMON Adaptiertes Monitoring. www.adamon.de

8 Menikoff J. The paradoxical problem with multiple-IRB review. N Engl J Med. 2010; 363:1591-3 gesetzt werden. Auch Projekte wie Therapieoptimierungsstudien mit bereits registrierten Medikamenten, Lebensqualitätsforschung und Untersuchungen zur Gesundheitsökonomie, die im Rahmen einer klinischen Studie durchgeführt werden, sind in ICH-GCP nicht abgebildet. Wenn ICH-GCP in diesen Bereichen eingesetzt wird, ist die Gefahr gross, dass unnötiger Aufwand entsteht.

Viele Studien mit kleinen Populationen, z.B. in der Pädiatrie oder bei seltenen Erkrankungen, werden auf Grund des hohen Aufwandes für den Sponsor und die Prüfärzte bei einer strikten Auslegung von ICHGCP nicht mehr in Angriff genommen. Diese Entwicklung läuft langfristig den Interessen der Patienten entgegen. Es sollte berücksichtigt werden, dass der medizinische Fortschritt in der nahen Zukunft insbesondere durch die Untersuchung von kleinen Populationen, die aufgrund von prädiktiven Markern eine Einheit bilden, vorwärtsgetrieben wird [5]. Um die unsystematischen individuellen Heilversuche einzudämmen, die bei Fehlen von wissenschaftlicher Evidenz häufig angewendet werden, sollten seltene Erkrankungen möglichst im Rahmen von klinischen Studien behandelt werden.

Akademische Institutionen wie die SAKK oder die Clinical Trial Units (CTUs) der Schweizer Spitäler können klinische Studien unter ICH-GCP durchführen, aber nur um den Preis erhöhter finanzieller und personeller Ressourcen. Die momentane Überregulierung reduziert die Produktivität der patientenorientierten klinischen Forschung und führt damit zu einer Verlangsamung des therapeutischen Fortschrittes $[3,4,5]$, ohne dass aus unserer Sicht ein zusätzlicher Beitrag zu den Zielen von GCP erreicht wird.

\section{Ansprüche an die zukünftige gesetzliche Regelung}

Unsere Institutionen stehen zu den GCP-Grundsätzen, wie sie z. B. im Kapitel 2.0 Principles of ICH-GCP formuliert sind. Eine Diskussion, wie diese Grundsätze z. B. im Rahmen der Therapieoptimierung mit bereits registrierten Arzneimitteln umzusetzen sind, sollte stattfinden. Weil die patientenorientierte klinische Forschung mehr als das Entwickeln von Medikamenten umfasst, sollten auch die anderen Forschungsbereiche adäquat geregelt werden. Diese Ausführungsbestimmungen müssen zwingend im Einklang mit den europäischen Entwicklungen stehen [6].

Solange die Behörden gesetzlich für die Beurteilung von allen Studien mit Medikamenten an ICHGCP gebunden sind, schlagen wir eine risikoadaptierte Auslegung vor. Die Auslegungsspielräume sollten so genutzt werden, dass die angestrebten Ziele mit möglichst geringem Aufwand erreicht werden. Damit dies realisierbar ist, braucht es beim Sponsor und den Prüfärzten, aber auch bei den Behörden Ausbildung, Erfahrung, Verständnis und Vertrauen sowie das richtige Augenmass für das realistische Risiko. Entsprechende Diskussionen, die zurzeit in der EU geführt werden [6, 7], sollten auch in der Schweiz aufgenommen werden. Es ist höchste Zeit zu spezifizieren, welche Detailanforderungen aus ICH-GCP für die Anwendung in der patientenorientierten Forschung übernommen werden und welche unter bestimmten Bedingungen weggelassen werden können. Durch eine risikoadaptierte Umsetzung der GCP-Grundsätze könnten mehr relevante Fragestellungen beantwortet werden.

Zwischen den Ethikkommissionen und Swissmedic sollten endlich klare Zuständigkeiten bei der Beurteilung von Forschungsprojekten hergestellt werden. Multiple Entscheide müssen in der Zukunft vermieden werden, da diese die Qualität der Forschung beeinträchtigen [8].

Wir fordern daher die Bundesbehörden auf, die Verantwortung für die Umsetzung von Artikel 1, Abs. 3 b, des Heilmittelgesetzes (HMG) zu übernehmen, der vorsieht, günstige Rahmenbedingungen für die Forschung im Heilmittelbereich zu schaffen. Dieser Grundsatz sollte auch im Bundesgesetz über die Forschung am Menschen (Humanforschungsgesetz) berücksichtigt werden. Vorab gilt es zu vermeiden, dass die ICH-GCP-Richtlinie im Humanforschungsgesetz verankert wird. Bei der Regulierung von Studien in seltenen Erkrankungen sollte das heutige System grundlegend überarbeitet werden und ein deutlicher Abbau der administrativen Aufwendungen erfolgen. Sind diese Forderungen erreicht, können sich die Forschenden wieder vermehrt auf ihre Kernaufgabe konzentrieren, nämlich gute Forschung zum Wohle der Patienten zu betreiben. 